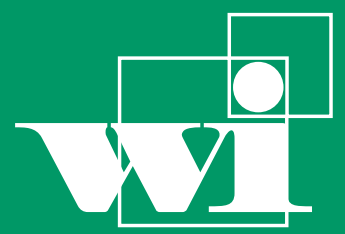

Bayreuther Arbeitspapiere zur Wirtschaftsinformatik

Jens Dibbern, Armin Heinzl

Outsourcing der Informationsverarbeitung im Mittelstand:

Test eines multitheoretischen Kausalmodells

Bayreuth Reports on Information Systems Management 
Die Arbeitspapiere des Lehrstuhls für Wirtschaftsinformatik dienen der Darstellung vorläufiger Ergebnisse, die i. d. R. noch für spätere Veröffentlichungen überarbeitet werden. Die Autoren sind deshalb für kritische Hinweise dankbar.

Alle Rechte vorbehalten. Insbesondere die der Übersetzung, des Nachdruckes, des Vortrags, der Entnahme von Abbildungen und Tabellen auch bei nur auszugsweiser Verwertung.

Authors:

Jens Dibbern

Armin Heinzl
The Bayreuth Reports on Information Systems Management comprise preliminary results which will usually be revised for subsequent publications. Critical comments would be appreciated by the authors.

All rights reserved. No part of this report may be reproduced by any means, or translated.

\section{Information Systems Management Working Paper Series}

\section{Edited by:}

Prof. Dr. Armin Heinzl

\section{Managing Assistant and Contact:}

Universität Bayreuth

Lehrstuhl für Wirtschaftsinformatik (BWL VII)

Prof. Dr. Torsten Eymann

Universitätsstrasse 30

95447 Bayreuth

Germany

Email: wi@uni-bayreuth.de
ISSN $1864-9300$ 


\section{Outsourcing der Informationsverarbeitung im Mittelstand: Test eines multitheoretischen Kausalmodells}

Jens Dibbern, Armin Heinzl

Arbeitspapier 9/2000

(Fassung vom 08.09.2000)

\section{Arbeitspapiere Wirtschaftsinformatik}

Herausgeber: Prof. Dr. Armin Heinzl

\section{Universität Bayreuth}

Lehrstuhl für Betriebswirtschaftslehre VII (Wirtschaftsinformatik)

Universitätsstraße 30, D-95440 Bayreuth

Telefon 0921/55-2807, Telefax 0921/55-2216

E-Mail:wi@uni-bayreuth.de

Internet: http://wi.oec.uni-bayreuth.de 


\title{
Outsourcing der Informationsverarbeitung im Mittelstand: Test eines multitheoretischen Kausalmodells
}

\begin{abstract}
Zusammenfassung:
In dem vorliegenden Beitrag werden aus der Transaktionskostentheorie, der „Resource-based Theory“ und der „Power Theory“ Determinanten des Outsourcing der IV abgeleitet und zu einem theoretischen Bezugsrahmen zusammengefasst. Dieser wird anhand von 34 mittelständischen Unternehmen einem Test unterzogen.
\end{abstract}

\section{Stichworte:}

Outsourcing der Informationsverarbeitung, KMU, Mittelstand, Transaktionskostentheorie, Resource-based Theory, Power Theory, Kausalanalyse, Structural Equation Modeling, Partial Least Squares

\begin{abstract}
:
In this paper determinants of IS outsourcing are deduced from transaction cost theory, resource-based theory and power theory. They are summarized in a theoretical framework which is tested using a sample of 34 small and medium sized firms.
\end{abstract}

\section{Keywords:}

Information systems outsourcing, small and medium sized firms, transaction cost theory, resource-based theory, power theory, structural equation modeling, partial least squares, empirical study 


\section{Kernpunkte für das Management:}

In dem vorliegenden Beitrag wird die Situation des Outsourcing der Informationsverarbeitung (IV) in einer für den Mittelstand spezifischen Branche beleuchtet. Auf Basis theoretischer Überlegungen werden die wesentlichen Determinanten des Outsourcing der IV abgeleitet und zu einem theoretischen Bezugsrahmen zusammengefasst. Dieser wird anhand von 34 kleinen und mittleren Unternehmen (KMU) einem empirischen Test unterzogen. Die wesentlichen Ergebnisse der Studie lassen sich wie folgt darstellen:

- Insgesamt kann der Anteil des Outsourcing der IV in KMU als gering eingestuft werden.

- Die Funktionen der IV werden in KMU selektiv, d.h. in unterschiedlichem Maße ausgelagert. Während die Anwendungsentwicklung den höchsten Anteil ausmacht, verbleibt die Planung und das Management weitgehend innerhalb der Unternehmensgrenzen.

- Die Einschätzung interner Ressourcendefizite gegenüber externen Dienstleistern bildet die dominierende Determinante zur Erklärung des unterschiedlichen Grades der Auslagerung von Funktionen der Informationsverarbeitung.

- Demgegenüber scheinen Rationalitätskalküle wie die Berücksichtigung von Transaktionskosten und die Einschätzung der strategischen Bedeutung der IV in den Hintergrund zu rücken. 


\section{Einleitung}

Vor 13 Jahren stellte Dearden [Dear87] die These auf, dass die innerbetriebliche Informationsverarbeitung verschwinden werde . Er führte an, dass externe Anbieter in der Lage seien, sich auf bestimmte Dienstleistungen im Bereich der Informationsverarbeitung (IV) zu spezialisieren. Sie würden dadurch sowohl Qualitäts- als auch Kostenvorteile gegenüber der Selbsterstellung dieser Leistungen im Unternehmen erzielen. Insofern sei der Übergang interner IVFunktionen auf ein rechtlich selbständiges Dienstleistungsunternehmen allein eine Frage der Zeit, bis sich der externe Anbietermarkt etabliert habe. Von dieser Auslagerungs- bzw. Outsourcing-Tendenz seien allein Unternehmen in bestimmten Branchen mit großen Kapazitäten in der Informationsverarbeitung auszuklammern. Für sie prophezeite er eine zunehmende Verselbstständigung der IV-Abteilung in Form von Profit Centers, die kapitalmäßig mit der Unternehmung verbunden seien (z. B. Tochter- oder Beteiligungsgesellschaften). Bezüglich dieser Organisationsform wird im Gegensatz zur Auslagerung von Ausgliederung gesprochen [Hein93; HeSt93].

Zieht man aus heutiger Sicht Bilanz, so lassen sich folgende Beobachtungen bzw. Phänomene für den deutschen Markt festhalten:

Der Anstieg des Outsourcing der Informationsverarbeitung verlief in den vergangenen zehn Jahren im Vergleich zu angloamerikanischen Ländern eher zögerlich ${ }^{1}$. Zwar sind in Deutschland in den letzten Jahren ebenfalls Zuwachsraten im Schwankungsbereich von ca. 10 bis 20 \% pro Jahr zu verzeichnen [IDC99], diese basieren allerdings auf einem niedrigeren Ausgangsniveau als beispielsweise in den USA, wo „Megadeals“ (z. B. Kodak mit IBM, DEC und Businessland oder Xerox mit EDS) Anfang der 90er Jahre für großes Aufsehen sorgten [MeKn98, S. 19]. Eine relativ starke Ausprägung erfährt in Deutschland die Ausgliederung der betrieblichen Informationsverarbeitung (z. B. Debis ehemals Tochtergesellschaft von Daimler-Chrysler, nun zur Deutschen Telekom gehörend) [Hein93]. Allerdings beschränkt sich die Wahl dieser organisatorischen Verankerung der Informationsverarbeitung der Unternehmung entsprechend der von Dearden aufgestellten Hypothese vornehmlich auf Großunternehmen. Eine 1997 durchgeführte Marktforschungsstudie von MC-Team Management Consulting zeigt auf, dass derzeit ca. 500 Unternehmen diese organisatorische Verankerung wählen, wobei es Anzeichen dafür gibt, dass aufgrund des zunehmenden Wettbewerbs mittelfristig nur $50 \%$ der ausgegliederten Bereiche überleben werden [Dern97]. Nur wenige Erkenntnisse konnten bislang bzgl. des Outsourcing-Verhaltens kleiner und mittlerer Unternehmen (KMU) gewonnen werden. Es kann allerdings festgehalten werden, dass von einem Ver- 
schwinden der Inhouse-Informationsverarbeitung keine Rede sein kann. Vielmehr läßt sich ein von Dearden unberücksichtigtes Phänomen beobachten: selektives Outsourcing. Hiermit ist gemeint, dass Unternehmen in den seltensten Fällen ihre gesamte Informationsverarbeitung auslagern, sondern lediglich bestimmte IV-Funktionen ganz oder teilweise an Externe auslagern. Dieses Phänomen wurde in einer Vielzahl empirischer Untersuchungen im angloamerikanischen Sprachraum nachgewiesen [GrCT94; LaHi94; LaWF96; SoAp95]. In einer ersten deskriptiven interkulturellen Studie konnten Apte et al. [ASHS97] aufzeigen, dass der jeweilige Anteil der Auslagerung verschiedener IV-Funktionen nicht nur in den USA, sonden auch in Japan und in Finnland unterschiedlich ausfällt. Einer europaweiten Studie von IDC [IDC98] zufolge lässt sich das Outsourcingverhalten in Deutschland in besonderem Maße durch eine selektive Vorgehensweise charakterisieren.

Auf Basis der beobachteten Phänomene sollen folgende Untersuchungsfragen beantwortet werden:

1. In welchem Ausmaß werden einzelne IV-Funktionen von KMU ausgelagert?

2. Von welchen Determinanten hängt der Grad des Outsourcing von IV-Funktionen in KMU $\mathrm{ab}$ ?

Zunächst wird ein theoretischer Bezugsrahmen entwickelt, der die Determinanten des Outsourcingverhaltens von Unternehmen hypothetisiert. Anschließend wird der Bezugsrahmen in ein Kausalmodell transformiert und anhand einer Stichprobe von KMU einem empirischen Test unterzogen.

\section{Theoretischer Bezugsrahmen}

Auf Basis einer Analyse der Theorie der Unternehmung können die Transaktionskostentheorie und die „Resource-based Theory“ als komplementäre Erklärungsansätze des selektiven IV-Outsourcing herausgearbeitet werden $\left[\mathrm{DiHeGü]} .^{2}\right.$ Beide Theorien haben gemeinsam, dass sie dem Management eine Rationalität unterstellen, die - wenngleich begrenzt rational oder erfahrungsbasiert - auf ein Gewinn- oder Wirtschaftlichkeiktsstreben ausgerichtet ist. Dieser Einseitigkeit des Rationalitätskalküls soll durch die Berücksichtigung der „Power Theory“ [Pfef81] eine Perspektive entgegengestellt werden, welche die Interessen des IV-Bereichs unabhängig von übergeordneten Unternehmenszielen reflektiert. Bereits 1969 hat Heinrich [Hein69] die Auffassung vertreten, dass auch die Bewertung nichtquantifizierbarer Ziele wie Vergrößerung von Macht in der Bewertung bei der Wahl Eigenfertigung oder Fremdbezug der IV zu berücksichtigen ist. Lacity und Hirschheim [LaHi93] haben in ihrer interpretativen 
Fallstudienuntersuchung für die Relevanz des Aspekts der Macht beim Outsourcing der IV Bestätigung gefunden. Der Machtaspekt soll daher in die Entwicklung des theoretischen Bezugsrahmens mit einfließen, wenngleich er nur begrenzt Aussagen bzgl. des selektiven Outsourcing zulässt, sondern eher bei der aggregierten Sichtweise - also der Betrachtung des gesamten IV-Bereichs einen alternativen Erklärungsansatz für unterschiedliches Outsourcingverhalten bietet. Die aus den einzelnen Theorien abgeleiteten Konstrukte und deren Beziehungen veranschaulicht die folgende Grafik:

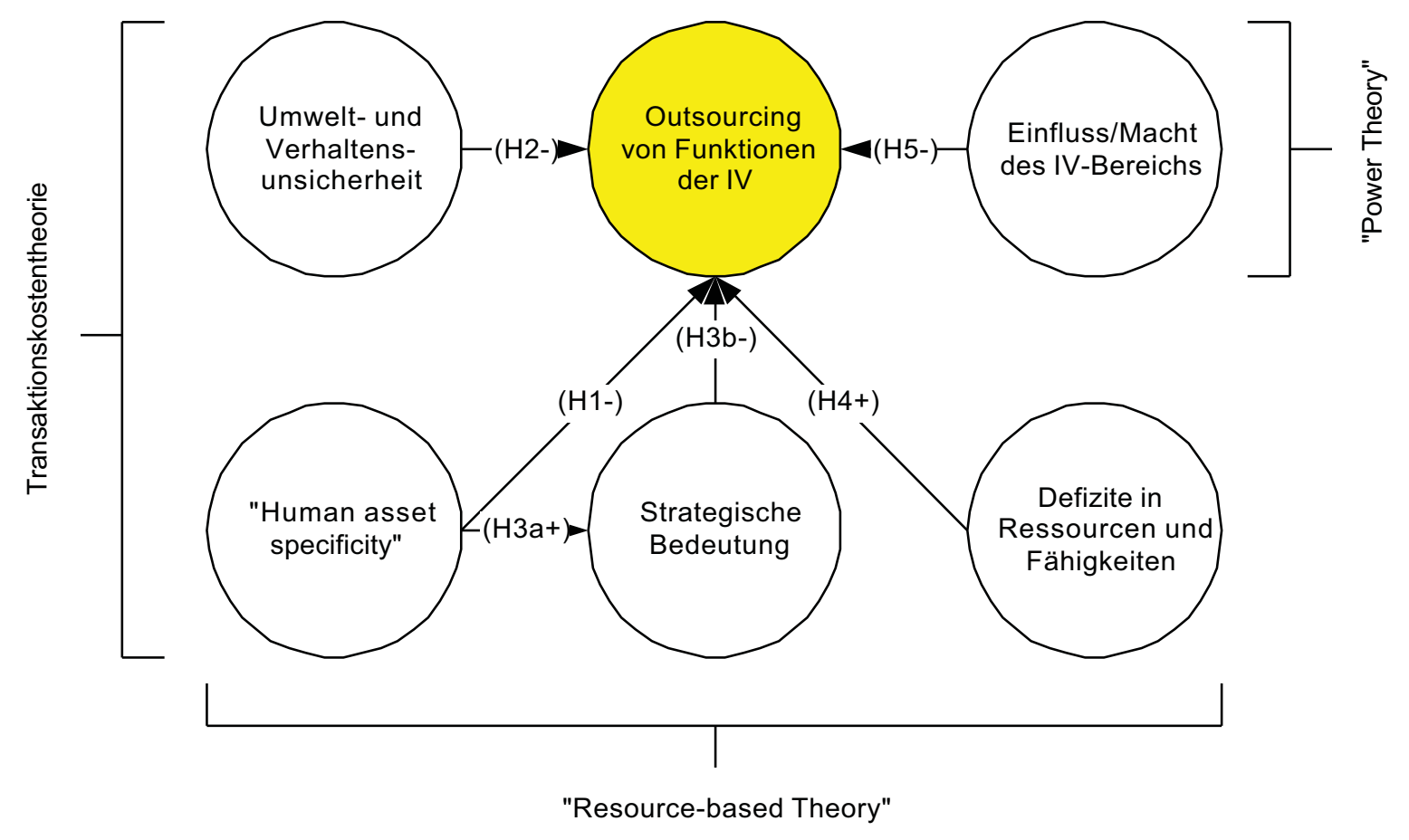

Bild 1 Theoretischer Bezugsrahmen des Outsourcing der Informationsverarbeitung

\subsection{Transaktionskostentheorie}

Die Einflussgrößen Umwelt- und Verhaltensunsicherheit sowie „human asset specificity“ entstammen der Transaktionskostentheorie. Nach der Transaktionskostentheorie entscheidet sich die Frage Eigenerstellung oder Fremdbezug (bzw. Markt versus Hierarchie) aufgrund eines Kostenvergleichs unter Berücksichtigung der Summe aus Transaktions- und Produktionskosten [Will75; ders. 81; 85; 90]. Im vorliegenden Beitrag sollen unter Transaktionskosten alle Kosten der Planung, Anpassung und Kontrolle der Aufgabenerfüllung in den einzelnen Funktionen der Informationsverarbeitung verstanden werden. Diese Kosten werden maßgeblich durch die Höhe der Umwelt- und Verhaltensunsicherheit sowie der „,asset specificity“ determiniert. 
Williamson [Will90, S. 142] zufolge bezeichnet die „,asset specificity“, inwiefern materielle oder immaterielle Wirtschaftsgüter außerhalb der Unternehmung den gleichen Mehrwert generieren können wie innerhalb der Unternehmung. Als materielle Wirtschaftsgüter sind im vorliegenden Kontext Hardwareprodukte, informations- und kommunikationstechnologische Infrastrukturen sowie vom Markt zu beziehende Standardsoftwareprodukte zu subsummieren. Sie weisen per se keine zwingenden Eigenschaften auf, die Unterschiede in der Ausprägung ihrer Spezifität begründen würden.

Bei der Definition immaterieller Wirtschaftsgüter bzw. der ,, human asset specificity” kann zwischen notwendigen und hinreichenden Bedingungen ihres Vorhandenseins unterschieden werden [Will81, S. 563]. Intellektuelle Fähigkeiten sowie autodidaktisch oder durch schulische Maßnahmen erlernbare Spezialkenntnisse (z.B. Programmierkenntnisse) der Arbeitnehmer sind ein notwendiges, aber kein hinreichendes Kriterium zur Beurteilung der Spezifität des Humankapitals einer Unternehmung. Ein hinreichendes Kriterium liefert die Antwort auf die Frage, inwieweit die Arbeitnehmer zur Verrichtung ihrer Aufgaben unternehmensinterne Kenntnisse benötigen, die sie nur durch einen ,learning-by-doing”-Prozess im Unternehmen erlangen können [Will81, S. 563]. Die IV ist als ,Querschnittsfunktion” durch die Spezifität des Humankapitals als inhärenter Eigenschaft der Teilfunktionen der IV gekennzeichnet. In besonderem Maße kommt diese Eigenschaft in solchen Teilfunktionen zum Tragen, die durch häufige bereichsübergreifende Aufgaben gekennzeichnet sind [Hein96, S. 280f.]. Diese schlagen sich organisatorisch insbesondere in der Häufigkeit funktionsübergreifender Team- und Projektarbeiten nieder [Krcm97, S. 243f.]. Es lässt sich argumentieren, dass bei IVFunktionen, die durch eine hohe Spezifität des Humankapitals gekennzeichnet sind, die Anbahnungs- und Anpassungskosten im Sinne von Kosten des Informationsaustausches bei einem externen Anbieter höher ausfallen als innerhalb der Unternehmung. Ein externer Anbieter muss erst die vielfältigen Beziehungsgeflechte und Interdependenzen seines Kunden verstehen lernen. Annahmegemäß wird ein externer Anbieter nicht bereit sein, diese spezifischen „Investitionen“ zu tätigen, da sie ihn daran hindern, Skalenerträge zu realisieren. Vielmehr wird er sich in diesem Fall opportunistisch verhalten, das heißt, er wird mit List jede Gelegenheit auf Kosten des Auftraggebers ausnutzen, seine spezifischen „Investitionen“ zu überkompensieren. Es lässt sich folgende Hypothese ableiten:

H1: Je höher der Grad der "human asset specificity" einer IV-Funktion einzustufen ist, desto weniger wird diese ausgelagert. 
Eine zweite wichtige Determinanten in der ,make-or-buy“-Entscheidung bildet aus transaktionskostentheoretischer Sicht die Unsicherheit. Einem begrenzt rationalen Entscheider ist es unmöglich, alle Eventualitäten in die Vertragsgestaltung mit einem externen Anbieter einfließen zu lassen. Zum Zeitpunkt der Vertragsgestaltung herrschen zum einen gewisse Informationsdefizite bezüglich zukünftiger sich ändernder Anforderungen an den Vertragsgegenstand, z.B. technologische Entwicklungen, zukünftige Anforderungen von Kunden und an Mitarbeiter, etc. (Umweltunsicherheit). Es lässt sich argumentieren, dass sich die zukünftigen Anforderungen an IV-Funktionen, die einem starken Einfluss durch technologischen Wandel und Innovationen ausgesetzt sind, in Verträgen mit externen Anbietern nur unpräzise spezifizieren lassen. Die Folge sind hohe Unsicherheiten in der Vertragsgestaltung und daraus resultierend mögliche hohe nachvertragliche Anpassungskosten [LaWF95]. Zum anderen können die Reaktionen des Vertragspartners in kritischen Situationen (Verhaltensunsicherheit), welche durch die genannten Umwelteinflüsse hervorgerufen werden können, nicht antizipiert werden. Die Verhaltensunsicherheit reflektiert das Potenzial der opportunistischen Verhaltensweise bei der Übernahme einer IV-Teilfunktion durch einen externen Dienstleister. Es lässt sich demnach folgende Hypothese ableiten:

H2: Je höher der Grad der Umwelt- und Verhaltensunsicherheit in Bezug auf eine IV-Funktion ausfällt, desto weniger wird diese ausgelagert.

\subsection{Resource-based Theory}

Die Transaktionskostentheorie unterstellt allerdings implizit, dass der Markt generell in der Lage ist, Sach- oder Dienstleistungen genauso gut herzustellen wie die Unternehmung selbst [Conn91; Dems91; Foss96; Lang95]. Die Unternehmung wird als Einheit ohne Vergangenheit betrachtet. Dieser statischen Sichtweise steht mit der „Resource-based Theory“ eine dynamisierte Betrachtung der Unternehmung ,entgegen” [Knud95]. Sie liefert Anhaltspunkte, wann Ressourcen unter strategischen Gesichtspunkten im Unternehmen behalten und ausgebaut und wann sie von einem externen Zulieferer bezogen werden sollten.

Entscheidend für diese Überlegungen ist einerseits die Analyse der zugrunde liegenden materiellen und immateriellen Ressourcen vor dem Hintergrund der Frage, ob diese anhaltende Wettbewerbsvorteile gegenüber Konkurrenten genieren können. Diese Voraussetzung ist insbesondere dann erfüllt, wenn es sich um unternehmensspezifische Ressourcen handelt, die nicht handelbar, imitierbar und substituierbar sind [DiCB89,S. 1507ff.]. 
Die in den einzelnen IV-Funktionen gebundenen physischen Ressourcen - sprich Hardware und Software - können per se nicht als unternehmensspezifisch eingeschätzt werden. Hardwarekomponenten und Standardsoftware können jederzeit vom Markt bezogen werden und sind vor Imitation nicht sicher [ClRo91; MaFB95]. Der fokussierende Blick ist also auf die in den einzelnen Funktionsbereichen enthaltenen immateriellen Ressourcen und Fähigkeiten zu richten. Zur Beurteilung der Spezifität gelten demnach die selben Überlegungen wie bei der „asset specificity”. Die Konstrukte spezifische „,assets“ und spezifische Ressourcen können daher als identisch behandelt werden [DiGüHe]. Es lässt sich folgende Hypothese aufstellen:

H3a: Je höher der Grad der „,human asset specificity“ einer IV-Funktion ausfällt, desto höher ist ihre strategische Bedeutung einzustufen.

Die Auslagerung einer Teilfunktion, in der Ressourcen und Fähigkeiten gebunden sind, die für eine Unternehmung von strategischer Bedeutung sind (,strategic assets“) macht wenig Sinn [DiCB89, S. 1505]. Zwar beinhaltet ein Outsourcingvertrag in der Regel, dass menschliche und physische Ressourcen einer Teilfunktion vom externen Zulieferer übernommen werden. Diese werden jedoch meist in einem neuen organisatorischen Umfeld zusammengeführt. Die Voraussetzungen für den Ausbau und die Neuentwicklung von unternehmensspezifischen Fähigkeiten bzw. Kernkompetenzen der Unternehmung sind somit eingeschränkt. Darüber hinaus ist davon auszugehen, dass der Outsourcinganbieter das in den übernommenen Ressourcen gebundene unternehmensspezifische Wissen an seine übrigen Kunden - unter denen sich möglicherweise direkte Konkurrenten des Vertragspartners befinden - weitergibt. Wettbewerbsvorteile gegenüber Konkurrenten können somit weder ausgebaut, noch aufrechterhalten werden. Die angeführten Überlegungen führen zu folgender Schlussfolgerung:

H3b: Je höher die strategische Bedeutung der Ressourcen und Fähigkeiten einer IV-Funktion ausfällt, desto weniger wird diese ausgelagert.

Bislang wurde davon ausgegangen, dass allein innerhalb der Unternehmensgrenzen ein Such- und Lernprozess stattfinden kann, der zum Ausbau und der Entwicklung strategisch wichtiger Ressourcen und Fähigkeiten führt. Damit wurde unterstellt, dass die interne Erstellung von Gütern und Dienstleistungen der externen stets überlegen ist. Diese Sichtweise vernachlässigt allerdings die Tatsache, dass auch der Markt in der Lage ist, aus Vergangenem zu lernen und aufgrund vorhandener Ressourcen eigene Fähigkeiten und Routinen zu entwickeln [Lang95, S. 92ff.]. Diese wiederum können von einer Unternehmung als komplementäre Ressourcen (,complementary assets") genutzt werden [TRDW94, S. 20f.]. Eine Unternehmung 
wird jedoch solange die marktlichen Kompetenzen nicht in Anspruch nehmen, wie sie die selben Fähigkeiten aufgrund interner Ressourcen selbst generieren kann. Werden in einer Unternehmung auf Basis ihrer strategischen Ausrichtung jedoch Defizite in Ressourcen und Fähigkeiten diagnostiziert, so wird der Markt zum Ausgleich dieser Defizite (,filling gaps”) interessant [Gran91, S. 131ff.]. Dabei wird der Markt umso attraktiver, je größer die Defizite und je geringer die eigenen Fähigkeiten zum Schließen der Lücken eingeschätzt werden. Übertragen auf die Frage der Auslagerung bestimmter Teilfunktionen der Informationsverarbeitung ergibt sich folgende Hypothese:

H4: Je größer die Defizite in unternehmenseigenen Ressourcen und Fähigkeiten einer IVFunktion ausfallen, desto eher wird diese ausgelagert.

\subsection{Power Theory}

Die „Power Theory“ kann als Ausprägung der verhaltenswissenschaftichen Theorie [CyMa63] verstanden werden, in der die Unternehmung als politische Koalition konzeptualisiert wird. Der Kern der Theorie liegt in der Erklärung der Beziehungen zwischen Interessen, Konflikten und Macht einzelner Individuen oder politischer Koalitionen in der Unternehmung [Morg95, S. 148].

Die Interessen der Individuen können sich innerhalb der Unternehmung durch die Bildung verschiedener Interessengruppen (politischer Koalitionen) widerspiegeln. Kommt es zu Interessenkonflikten, bildet die Macht (,,power”) und das politische Handeln (,,politics”) der einzelnen Parteien das Medium zur ultimativen Konfliktlösung. Macht kann definiert werden als das Potenzial einer Partei, das Verhalten einer anderen Partei in einer bestimmten Angelegenheit zu beeinflussen, während ,politics” die Art und Weise beinhaltet, wie Macht ausgeübt wird [Tush77, S. 207; Pfef81, S. 7]. Im Folgenden wird ausschließlich die Machtkomponente betrachtet.

Ein Unternehmensbereich, der eine hohe Machtstellung im Unternehmen innehat, wird bestrebt sein, diese nach Möglichkeit zu erhalten oder auszubauen. Mit jeder Ressource, die ein Unternehmensbereich aufgrund von Rationalisierungs-, Umstrukturierungs-, oder Outsourcingprozessen verliert, verringert sich dessen Machtpotenzial. Für einen Unternehmensbereich, der relativ wenig Macht im Unternehmen auf sich vereinigt, bestehen nur geringe Chancen, sich gegen einen solchen Prozess zur Wehr zu setzen. Oftmals bestehen für die Betroffenen jedoch gar keine Anreize zu einer restriktiven Verhaltensweise. Dieser Fall tritt insbesondere dann ein, wenn sie in ihrer neuen Position ein höheres Machtpotenzial vorfinden 
als in ihrer ursprünglichen. Die Karriereaussichten und Möglichkeiten der persönlichen Entfaltung im Unternehmen werden dann entsprechend höher eingeschätzt. Die Wahrscheinlichkeit einer solchen Konstellation ist umso höher, je niedriger die Macht des angestammten Unternehmensbereichs ausfällt. Bietet der angestammte Unternehmensbereich jedoch ein hohes Machtpotenzial, so ist die Wahrscheinlichkeit, dass man in der neuen Position besser gestellt ist als vorher, eher gering. In diesem Fall wird die Resistenz gegen eine Auslagerung entsprechend hoch sein und das Machtpotenzial im Unternehmen wird genutzt werden, um einen solchen Prozess zu verhindern oder gar nicht erst aufkommen zu lassen. Hinsichtlich der Auslagerungsentscheidung läßt sich nach der „Power Theory“ somit folgende Hypothese ableiten:

H5: Je größer die relative Macht der IV-Abteilung innerhalb der Unternehmung einzustufen ist, desto weniger werden Funktionen der IV ausgelagert.

\section{Untersuchungsmethodik}

\subsection{Datenerhebung}

Die Datenerhebung wurde mit Hilfe eines Fragebogens durchgeführt. Der Fragebogen wurde zunächst anhand von Praktikern auf seine inhaltliche Verständlichkeit geprüft und anschließend an $281 \mathrm{KMU}$ verschickt. Die Untersuchungsobjekte setzten sich aus Unternehmen in Bayern (113), Baden-Württemberg (95) und Hessen/Rheinland-Pfalz/Saarland (73) zusammen, die dem Branchensektor Glas, Boden, Erden und Keramik zugeordnet werden können. Als Adressat des Fragebogens wurde jeweils die Unternehmensführung angesprochen. Insgesamt wurden 34 auswertbare Fragebögen zurückgesandt, was einer Rücklaufquote von $12 \%$ entspricht. Um zu gewährleisten, dass die auswertbaren Fragebögen eine repräsentative Stichprobe darstellen, wurden die sofort antwortenden Unternehmen mit den erst in einer Nachfassaktion antwortenden Unternehmen anhand der Unternehmenscharakteristika Umsatz und Anzahl der Mitarbeiter verglichen. Es konnten keine signifikanten Abweichungen zwischen den beiden Gruppen festgestellt werden (t-Test, $\mathrm{p}<0,05)$.

\subsection{Messinstrumentarien}

Das Outsourcingverhalten als zentrale abhängige Variable der Untersuchung wird mit einer Frage gemessen, die den prozentualen Anteil des Outsourcing am Gesamtbudget der jeweiligen IV-Funktion erhebt. Tabelle 1 zeigt auf, welche Teilfunktionen unterschieden werden [in Anlehnung an MeKn98]. 


\begin{tabular}{|c|c|c|c|c|}
\hline $\begin{array}{l}\text { Systembetrieb / } \\
\text { Rechenzentrum }\end{array}$ & $\begin{array}{l}\text { Telekommunikation I } \\
\text { Netzwerke }\end{array}$ & $\begin{array}{l}\text { Anwendungsent- } \\
\text { wicklung, -einführung } \\
\text { und -pflege }\end{array}$ & $\begin{array}{l}\text { Information Center I } \\
\text { Benutzer Support }\end{array}$ & $\begin{array}{l}\text { IV-Planung und IV- } \\
\text { Management }\end{array}$ \\
\hline $\begin{array}{l}\text { Installation, Betrieb } \\
\text { und technische War- } \\
\text { tung zentraler und } \\
\text { dezentraler Rechner } \\
\text { (Client / Serversyste- } \\
\text { me) bzw. systemnaher } \\
\text { Software } \\
\text { Systemprogrammie- } \\
\text { rung } \\
\text { Systemsteuerung } \\
\text { Sicherheit und Katas- } \\
\text { trophenvorsorge }\end{array}$ & $\begin{array}{l}\text { Aufbau, Betrieb und } \\
\text { Wartung von Netzwer- } \\
\text { ken } \\
\text { Einbindung und Admi- } \\
\text { nistration von Daten- } \\
\text { und Anwendungsser- } \\
\text { vern in Netzwerke } \\
\text { Einführung und Betrieb } \\
\text { von entsprechenden } \\
\text { Diensten für den inner- } \\
\text { und überbetrieblichen } \\
\text { Informationsaustausch }\end{array}$ & $\begin{array}{l}\text { Eigene Software- und } \\
\text { Anwendungsentwick- } \\
\text { lung } \\
\text { Systemanalyse } \\
\text { Projektmanagement } \\
\text { Wartung bestehender } \\
\text { Anwendungen } \\
\text { Daten(bank)- } \\
\text { administration } \\
\text { Qualitätssicherung } \\
\text { Einführung und Anpas- } \\
\text { sung von Standard- } \\
\text { softwarepaketen (z. B. } \\
\text { SAP R/3) }\end{array}$ & $\begin{array}{l}\text { Beratung und Unter- } \\
\text { stützung der Benutzer } \\
\text { Schulung, Aus- und } \\
\text { Weiterbildung der } \\
\text { Benutzer } \\
\text { Problem-Management } \\
\text { Brückenfunktion zw ł } \\
\text { schen Fachbereichen } \\
\text { und IV-Bereich } \\
\text { Test, Beschaffung, } \\
\text { Installation, Einführung } \\
\text { und Wartung von } \\
\text { Hardware und Sof t- } \\
\text { ware }\end{array}$ & $\begin{array}{l}\text { Langfristige IV-Planung } \\
\text { Integration von Unter- } \\
\text { nehmensplanung und } \\
\text { IV-Planung } \\
\text { Grundsatzfragen } \\
\text { Identifizieren von } \\
\text { zukünftigen IV- } \\
\text { Innovationen } \\
\text { IV-Controlling } \\
\text { Konzeption von Sys- } \\
\text { temarchitekturen } \\
\text { Standards und Metho- } \\
\text { den }\end{array}$ \\
\hline
\end{tabular}

Tabelle 1 Funktionen der Informationsverarbeitung

Zur Charakterisierung der Stichprobe werden vier demographische Variablen herangezogen: Die Größe des Gesamtunternehmens wird anhand des Umsatz und der Anzahl der Mitarbeiter gemessen. Als Größenmerkmale der Informationsverarbeitung werden das IV-Budget und die Anzahl der IV-Mitarbeiter gewählt. Um Anhaltspunkte über die Gewichtung der einzelnen IV-Funktionen zu erlangen, wird zudem der prozentuale Anteil der einzelnen Funktionen am Gesamtbudget der Informationsverarbeitung erhoben.

Die unabhängigen Variablen werden bis auf das Unsicherheitskonstrukt mit Fragen bzw. Indikatoren gemessen, welche das jeweilige Konstrukt reflektieren. Aufgrund der Komplexität des Konstrukts der Verhaltens- und Umweltunsicherheit wird dieses im sogenannten formativen Modus operationalisiert [Chin98c; Forn89]. Diesem Modus liegt die Annahme zugrunde, dass die einzelnen Indikatoren unterschiedliche Ausprägungen des Konstrukts verursachen. Anders als im reflektiven Modus können die einzelnen Indikatoren, die zusammen das Messmodell einer Größe bilden, unabhängig voneinander sein. Als solche verursachenden Indikatoren werden - jeweils bezogen auf die einzelne IV-Funktionen - (1) die Anzahl geeigneter externer Anbieter, (2) die Höhe des Wandels der Anforderungen und Aufgaben und (3) die Schwierigkeit der Messbarkeit der Aufgabenerfüllung herangezogen.

Die ,, human asset specificity“ “wird daran gemessen, inwiefern die Mitarbeiter in den jeweiligen IV-Funktionensbereichen Kenntnisse (1) über die Arbeitsabläufe und Bedürfnisse der Fachabteilungen sowie (2) über die Beziehungen der Fachabteilungen untereinander und zu Kunden, Lieferanten oder sonstigen externen Bezugsgruppen benötigen und (3) inwiefern mit den Fachabteilungen zusammengearbeitet wird, z.B. in Form von Team- oder Projektarbeit. 
Die strategische Bedeutung der einzelnen IV-Funktionen wird anhand des Beitrags der einzelnen IV-Funktionen zum Erzielen von (1) Kostenvorteilen und (2) Wettbewerbsvorteilen durch Differenzierung des Leistungsspektrums gegenüber konkurrierenden Unternehmen gmessen.

Ressourcendefizite reflektieren, inwiefern die Fähigkeiten eines externen Dienstleisters, (1) vorhandene und (2) zukünftige Defizite bei der Erfüllung von Aufgaben in einzelnen IVFunktionen zu beheben, höher eingeschätzt werden als die eigenen.

Die Macht wird nicht auf die einzelnen IV-Funktionen, sondern auf den IV-Bereich als Ganzes bezogen. Sie wird gemessen indem die (1) Hierarchieebene der IV-Abteilung im Unternehmen und (2) der Grad der finanziellen Autonomie der IV-Abteilung abgefragt wird.

\subsection{Die Kausalanalyse}

Die Datenanalyse wurde mit Hilfe des komponenten-basierten statistischen Verfahrens zur Kausalanalyse „Partial Least Squares“ (PLS) durchgeführt [Chin98c; ChNe99]. Die Kausalanalyse („Structural Equation Modeling“) stellt ein statistisches Verfahren dar, mit dem theoretisch begründete Hypothesen überprüft werden können. Sie besitzt einen primär konfirmatorischem Charakter. Mit Hilfe der Kausalanalyse können sowohl direkte, als auch indirekte Beziehungen zwischen mehreren nicht direkt beobachtbaren Größen, sog. latenten Variablen bzw. hypothetischen Konstrukten, überprüft werden. Voraussetzung für die Messung von Zusammenhängen zwischen nicht beobachtbaren Größen ist die Transformation der theoretischen Sprache in eine Beobachtungssprache, d.h. die latenten Variablen sind zu operationalisieren. Die operationalisierten Größen stellen Messindikatoren dar, die in ihrer Summe ein Messmodell bilden. Die Beziehungen zwischen den nicht direkt beobachtbaren Größen und den beobachtbaren Messindikatoren werden als Korrespondenzhypothesen aufgefasst. Die Beziehungen aller Variablen bilden das Strukturmodell. Letzteres weist separat betrachtet dieselben Eigenschaften auf wie ein theoretischer Bezugsrahmen. Insofern kann Bild 1 auch als Strukturmodell des Outsourcing der IV aufgefasst werden.

Das PLS-Verfahren [Chin98c; ChNe99; Wold89] findet in den letzten Jahren neben den üblichen Verfahren des kovarianz-basierten „Structural Equation Modeling“ (z. B. LISREL, EQS, AMOS) zunehmend Verbreitung. Neben der größeren Flexibilität in der Modellierung der Messindikatoren (sowohl Modellierung reflektiver als auch formativer Indikatoren) ist die Attraktivität des Verfahrens unter anderem auf die geringen Anforderungen an die Stichprobengröße und die Unabhängigkeit vom Vorhandensein normal verteilter Daten zurückzufüh- 
ren. Da das Konstrukt der Verhaltens- und Umweltunsicherheit im formativen Modus operationalisiert wurde und die Stichprobengröße mit $n=34$ als gering einzustufen ist, wurde PLS als adäquates Analyseinstrumentarium erachtet. Die Signifikanztests für alle Pfade wurden mit Hilfe des ,bootstrap resampling“-Verfahrens durchgeführt [ChNe99]. Für die Beurteilung der Validität der reflektiven Messinstrumentarien wurde die Höhe der Faktorladungen herangezogen (höher als 0,7). Im Falle formativer Indikatoren wurden entsprechend der Empfehlung von Chin [Chin98c] die Gewichte der einzelnen Indikatoren beurteilt.

Im Rahmen der Analyse wurde das Modell einerseits unter Einbeziehung aller 5 IVFunktionen getestet. Aufgrund dieser Aggregation der Teilfunktionen wurde ein Stichprobenumfang von $\mathrm{n}=5 \times 34=170$ realisiert. $^{3}$ Andererseits wurde das Kausalmodell für jede der einzelnen IV-Funktionen $(\mathrm{n}=34)$ einem Test unterzogen, um $\mathrm{zu}$ untersuchen, inwiefern es Abweichungen bzgl. des Erklärungsgehalts des Modells zwischen den einzelnen IVFunktionen gibt.

\section{Empirische Befunde}

\subsection{Deskriptive Statistiken}

Die deskriptiven Ergebnisse der Studie beziehen sich auf die erstgenannte Forschungsfrage, die darauf abzielt, den Erkenntnisstand über das Ausmaß des IV-Outsourcing in mittelständischen Unternehmen zu erweitern. Zur besseren Einordnung der Ergebnisse werden zunächst die demographischen Charakteristika der Stichprobe anhand der folgenden Graphik veranschaulicht:

\begin{tabular}{|l|c|r|r|r|r|}
\hline Charakteristika & Anzahl & Minimum & Maximum & Mittelwert & Standarda bweichung \\
\hline Umsatz in Mill. DM & 31 & 15,00 & 750,00 & 133,91 & 161,09 \\
\hline \hline IV-Ausgaben/Umsatz & 29 & 0,10 & 1,80 & 0,70 & 0,44 \\
\hline Anzahl der Mitarbeiter & 33 & 25 & 1900 & 525 & 532,00 \\
\hline Anzahl der IV Mitarbeiter & 33 & 0 & 22 & 5 & 5,30 \\
\hline
\end{tabular}

\section{Tabelle 2 Demographische Charakteristika}

Es fällt auf, dass der Anteil der IV-Ausgaben am Gesamtumsatz der untersuchten KMU gring ausfällt (Mittelwert: 0,7\%). Auch die Anzahl der IV-Mitarbeiter ist im Durchschnitt mit fünf Mitarbeitern als gering einzustufen, was darauf hinweist, dass die Arbeitsteilung in der Informationsverarbeitung rudimentär ausgeprägt ist. 
Ein Blick auf den Grad des Outsourcing der einzelnen IV-Funktionen zeigt auf, dass die Beobachtung der geringen personalen Ressourcenausstattung der Informationsverarbeitung nicht auf einen hohen Outsouring-Anteil in den KMU zurückzuführen ist. Der Grad des Outsourcing kann mit Mittelwerten zwischen $6 \%$ und $31 \%$ als moderat eingestuft werden (vgl. Tabelle 3). Ob diese Beobachtungen ein Spezifikum der untersuchten Branche oder ein generelles Phänomen des Mittelstands sind, kann erst beurteilt werden, wenn Daten in anderen Branchen vorliegen.

\begin{tabular}{|l|r|r|r|r|}
\hline \multicolumn{1}{|c|}{ IV-Funktionen } & Anzahl & Maximum & Mittelwert & Standarda bweichung \\
\hline Systembetrieb / Rechenzentrum & \multicolumn{1}{|c|}{33} & 100,00 & $\mathbf{2 2 , 7 2}$ & 28,01 \\
\hline Telekommunikation / Netzwerke & 33 & 100,00 & $\mathbf{2 3 , 6 7}$ & 29,97 \\
\hline Anwendungsentwicklung, -einführung und -pflege & \multicolumn{1}{|c|}{33} & 90,00 & $\mathbf{3 1 , 1 2}$ & 32,31 \\
\hline Information Center / Benutzer Support & 33 & 90,00 & $\mathbf{1 7 , 7 6}$ & 22,47 \\
\hline IV-Planung und -Management & 33 & 50,00 & $\mathbf{6 , 1 2}$ & 10,49 \\
\hline
\end{tabular}

Tabelle 3 Outsourcinganteil pro IV-Funktion

Den relativ größten durchschnittlichen Outsourcinganteil weist die Anwendungsentwicklung und -wartung auf (Mittelwert: $31 \%$ ), gefolgt von den Teilfunktionen Telekommunikation / Netzwerke, Systembetrieb / Rechenzentrum, Benutzersupport und IV-Planung / Management. Die relativen Anteile der IV-Ausgaben der einzelnen IV-Funktionen am Gesamtbudget der IV zeigen auf, dass der Systembetrieb den höchsten durchschnittlichen Ausgabenanteil aufweist (30\%), gefolgt von Anwendungsentwicklung (27\%), Telekommunikation / Netzwerke (18 $\%)$, Benutzersupport (15\%) und IV-Management (10\%).

In einer Kontrollfrage konnte zudem ein hoher Anteil an fremdbezogener Standardsoftware der antwortenden Unternehmen festgestellt werden. Bei näherer Betrachtung zeigt sich, dass die KMU insbesondere in den betriebswirtschaftlichen Funktionsbereichen auf Standardsoftware-Pakete zurückgreifen. In den Bereichen Finanz- und Rechnungswesen setzen 76 \% der befragten Unternehmen ein Standardsoftware-Produkt ein, wobei die Pakete SAP R/3 und COMET von Siemens die Vormachtstellung einnehmen. Weniger verbreitet ist der Einsatz von Standardsoftware in den Bereichen Logistik (32 \%) und Personal (29\%).

\subsection{Test des Kausalmodells}

Der Test des Kausalmodells bezieht sich auf die zweite Forschungsfrage, die nach den Determinanten des Outsourcing von IV-Funktionen fragt. Bei einer ersten Analyse des aggre- 
gierten Modells und der Einzelmodelle wurde sichtbar, dass die drei Indikatoren des Konstrukts Umwelt- und Verhaltensunsicherheit teilweise mit unterschiedlichen Gewichten und Vorzeichen zu dem Konstrukt in Beziehung stehen. Aufgrund dieser Ambivalenz werden die einzelnen Messinstrumente im Folgenden als separate Konstrukte behandelt. Die ursprünglichen Korrespondenzhypothesen werden in Hypothesen trans formiert:

H2a: Je geringer die Anzahl geeigneter externer Anbieter ausfällt, desto weniger wird eine IVFunktion ausgelagert.

H2b: Je höher der Wandel der Anforderungen und Aufgaben der einzelnen IV-Funktionen, desto weniger werden diese ausgelagert.

H2c: Je größer die Schwierigkeiten der Messbarkeit der Aufgabenerfüllung der einzelnen IVFunktionen, desto weniger werden diese ausgelagert.

Auf diese Weise werden die einzelnen Beziehungen der Indikatoren, die nun jeweils eine eigenständige Einflussgröße verkörpern, besser sichtbar und falsifizierbar. Es sei allerdings angemerkt, dass es sich hierbei um eine Veränderung der ursprünglichen Modellstruktur im Sinne einer Exploration im konfirmatorischen Untersuchungsdesign handelt [Chin98a; LeBW97, S. 121].

Die Ergebnisse des modifizierten Modells werden in graphischer Form exemplarisch anhand des Einzelmodells der IV-Funktion Anwendungsentwicklung, -einführung und pflege dargestellt (vgl. Bild 2). Die Testergebnisse jeder einzelnen Hypothese sind den jeweiligen Pfaden zugeordnet. Die Pfadkoeffizienten (standardisiert) sind zusammen mit den jeweiligen tWerten abgebildet. Alle signifikanten Pfade sind je nach Signifikanzniveau mit einem Sternchen $(p<0,10)$, mit zwei $(p<0,05)$ oder mit drei $(p<0,01)$ Sternchen versehen.

Es zeigt sich, dass sich allein die Hypothesen H3a+ (starker positiver Zusammenhang zwischen „human asset specificity“ und strategischer Bedeutung) und H4+ (gemäßigt starker positiver Zusammenhang zwischen Ressourcendefiziten und Grad des IV-Outsourcing) bestätigen. Bei den Hypothesen $\mathrm{H} 2 \mathrm{a}+$ und H2c- kann jeweils ein umgekehrter signifikanter Zusammenhang festgestellt werden. Als nicht signifikant erweisen sich die Hypothesen H1-, H2bund H5-. Insgesamt werden 38,2 \% $\left[\mathrm{R}^{2}(\mathrm{Out})=0,382\right]$ der Varianz der abhängigen Variablen - Grad des IV-Outsourcing - durch das Modell erklärt. 


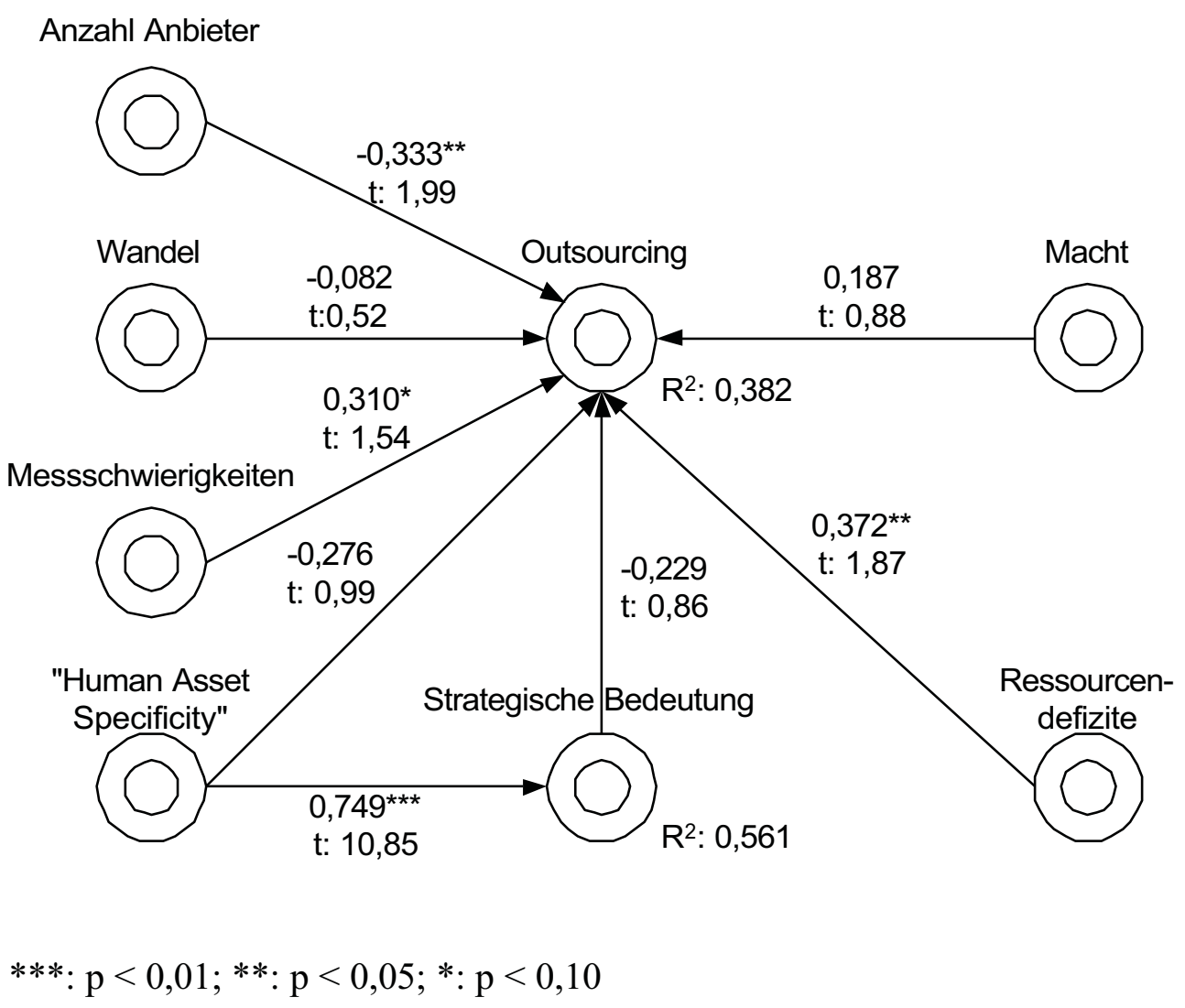

Bild 2 Kausalmodell des Outsourcing der IV-Funktion Anwendungsentwicklung, -einführung und -pflege

Die Ergebnisse aller Modelle veranschaulicht die folgende Tabelle:

\begin{tabular}{|c|c|c|c|c|c|c|c|c|c|c|}
\hline \multirow[t]{2}{*}{ IV-Funktionen } & \multicolumn{8}{|c|}{ Hypothesen } & \multicolumn{2}{|c|}{$\mathbf{R}^{2}$} \\
\hline & $\begin{array}{l}\text { H1(-) } \\
\text { Asset }\end{array}$ & $\begin{array}{l}\mathrm{H} 2 \mathrm{a}(+) \\
\mathrm{Anz}\end{array}$ & $\begin{array}{l}\mathrm{H} 2 \mathrm{~b}(-) \\
\text { Wan }\end{array}$ & $\begin{array}{l}\text { H2c(-) } \\
\text { Mess }\end{array}$ & $\begin{array}{l}\mathrm{H} 3 \mathrm{a}(+) \\
\text { Ass-Str }\end{array}$ & $\begin{array}{l}\mathrm{H} 3 \mathrm{~b}(-) \\
\text { Strat }\end{array}$ & $\begin{array}{l}\mathrm{H} 4(+) \\
\text { Ress }\end{array}$ & $\begin{array}{l}\text { H5(-) } \\
\text { Pow }\end{array}$ & Strat & Out \\
\hline $\begin{array}{l}\text { Anwendungsentwicklung, } \\
\text {-einführung und -pflege }\end{array}$ & $\begin{array}{l}-0,28 \\
\mathrm{t}: 0,99\end{array}$ & $\begin{array}{l}-0,33 * * \\
\mathrm{t}: 1,99\end{array}$ & $\begin{array}{l}0,08 \\
\text { t: } 0,52\end{array}$ & $\begin{array}{l}0,31^{*} \\
\mathrm{t}: 1,54\end{array}$ & $\begin{array}{l}0,75^{* * *} \\
\mathrm{t}: 10,85\end{array}$ & $\begin{array}{l}-0,23 \\
t: 0,86\end{array}$ & $\begin{array}{l}0,37 * * \\
\text { t: } 1,87\end{array}$ & \begin{tabular}{|l|}
0,19 \\
t: 0,88
\end{tabular} & 0,561 & 0,382 \\
\hline $\begin{array}{l}\text { Systembetrieb / Rechen- } \\
\text { zentrum }\end{array}$ & $\begin{array}{l}0,030 \\
\mathrm{t}: 0,12\end{array}$ & $\begin{array}{l}-0,07 \\
\mathrm{t}: 0,10\end{array}$ & $\begin{array}{l}-0,30^{*} \\
\mathrm{t}: 1,38\end{array}$ & $\begin{array}{l}0,54^{* *} \\
\mathrm{t}: 2,52\end{array}$ & $\begin{array}{l}0,355 \\
\mathrm{t}: 0,12\end{array}$ & $\begin{array}{l}0,083 \\
\text { t: } 0,49\end{array}$ & $\begin{array}{l}0,26^{*} \\
\mathrm{t}: 1,61\end{array}$ & $\begin{array}{l}0,26 \\
\mathrm{t}: 1,05\end{array}$ & 0,126 & 0,439 \\
\hline $\begin{array}{l}\text { Information Center / } \\
\text { Benutzer Support }\end{array}$ & $\begin{array}{l}0,28 \\
\mathrm{t}: 1,03\end{array}$ & $\begin{array}{l}-0,29^{* *} \\
\text { t: } 1,77\end{array}$ & $\begin{array}{l}0,10 \\
\text { t: } 0,62\end{array}$ & $\begin{array}{l}0,41^{* * *} \\
\mathrm{t}: 2,68\end{array}$ & $\begin{array}{l}0,61^{* * *} \\
\mathrm{t}: 6,28\end{array}$ & $\begin{array}{l}0,37^{*} \\
\text { t: } 1,43\end{array}$ & $\begin{array}{l}0,44^{* * *} \\
\mathrm{t}: 2,97\end{array}$ & $\begin{array}{l}0,48^{* *} \\
\mathrm{t}: 2,14\end{array}$ & 0,380 & 0,454 \\
\hline $\begin{array}{l}\text { Telekommunikation / } \\
\text { Netzwerke }\end{array}$ & $\begin{array}{l}0,058 \\
\mathrm{t}: 0,17\end{array}$ & $\begin{array}{l}-0,26^{*} \\
\mathrm{t}: 1,52\end{array}$ & $\begin{array}{l}0,24^{*} \\
\mathrm{t}: 1,46\end{array}$ & $\begin{array}{l}-0,13 \\
t: 0,55\end{array}$ & $\begin{array}{l}0,43^{*} \\
\mathrm{t}: 1,54\end{array}$ & $\begin{array}{l}-0,28 \\
t: 1,00\end{array}$ & $\begin{array}{l}0,18 \\
\text { t: } 0,88\end{array}$ & $\begin{array}{l}-0,22 \\
t: 0,86\end{array}$ & 0,189 & 0,206 \\
\hline Planung und Management & $\begin{array}{l}0,22 \\
\mathrm{t}: 0,61 \\
\end{array}$ & $\begin{array}{l}-0,08 \\
\text { t: } 0,36 \\
\end{array}$ & $\begin{array}{l}0,13 \\
\mathrm{t}: 0,49 \\
\end{array}$ & \begin{tabular}{|l|}
0,12 \\
$\mathrm{t}: 0,47$ \\
\end{tabular} & $\begin{array}{l}0,41 * * * \\
\mathrm{t}: 2,56 \\
\end{array}$ & $\begin{array}{l}-0,28 \\
\mathrm{t}: 0,94 \\
\end{array}$ & $\begin{array}{l}0,21 \\
\mathrm{t}: 1,03 \\
\end{array}$ & \begin{tabular}{|l|}
0,27 \\
$\mathrm{t}: 1,16$ \\
\end{tabular} & 0,164 & 0,277 \\
\hline $\begin{array}{l}\text { Aggregation aller IV- } \\
\text { Funktionen }\end{array}$ & $\begin{array}{l}0,01 \\
\mathrm{t}: 0,00\end{array}$ & $\begin{array}{l}0,05 \\
\text { t: } 0,45\end{array}$ & $\begin{array}{l}0,20 \\
t: 1,23\end{array}$ & \begin{tabular}{|l}
$0,21 *$ \\
1,30
\end{tabular} & $\begin{array}{l}0,56^{* * *} \\
t: 10,15\end{array}$ & $\begin{array}{l}0,17^{*} \\
\mathrm{t}: 1,36\end{array}$ & $\begin{array}{l}0,37 * * * \\
\text { t: } 5,42\end{array}$ & \begin{tabular}{|l}
$0,16^{* * *}$ \\
1,93
\end{tabular} & 0,318 & 0,218 \\
\hline
\end{tabular}

Tabelle 4 Pfadkoeffizienten und $t$-Werte $\left(^{* * *}: p<0,01\left[t_{\text {crit }}=2,390\right]\right.$; ${ }^{* *}: p<0,05\left[t_{\text {crit }}=1,671\right]$; *:

$$
\left.\mathrm{p}<0,10\left[\mathrm{t}_{\text {crit }}=1,296\right]\right)
$$

Die Faktorladungen betragen in allen sechs Modellen - mit drei Ausnahmen - Werte über 0,7 bei einem Mindestsignifikanzniveau von $\mathrm{p}<0,05$, wobei die meisten Ladungen Werte über $0,8(\mathrm{p}<0,01)$ erreichen. Allein bei der IV-Funktion Planung und Management kann eine 
nicht signifikante Faktorladung des Machtindikators Finanzielle Unabhängigkeit festgestellt werden. Bei der IV-Funktion Telekommunikation / Netzwerke erweisen sich der Indikator der „human asset specificity“ Kenntnisse der Fachabteilungen sowie der Machtindikator Hierarchieebene als instabil. Den Konstrukten Anzahl der Anbieter, Wandel, Messschwierigkeiten und Grad des IV-Outsourcing ist jeweils nur ein Indikator zugeordnet. Dementsprechend sind die Faktorladungen von der Parameterschätzung ausgeno mmen und auf 1,0 fixiert.

\section{Diskussion}

Im Rahmen der Diskussion wird eine Interpretation der zugrunde liegenden Ergebnisse vorgenommen. Generell lässt sich festhalten, dass sich die aus der Transaktionskostentheorie abgeleiteten Begründungszusammenhänge nicht bestätigten. Diese beruhen auf der Annahme, dass die interne Verankerung von IV-Funktionen bzw. deren vertikale Integration auf dem Kalkül der Beurteilung von Marktversagen beruht, d.h. der Frage, wann - unter Berücksichtigung von Transaktions- und Produktionskosten - externe Anbieter Effizienznachteile gegenüber der internen Erstellung einer bestimmten Teilfunktion aufweisen. Die Ergebnisse der Studie lassen schließen, dass bei den untersuchten mittelständischen Unternehmen derartigen Überlegungen keine oder nur eine untergeordnete Rolle bei der Wahl Markt vs. Hierarchie zukommt. Als konkurrierendes Rationalitätskalkül wurde das der strategischen Bedeutung der einzelnen IV-Funktionen herangezogen. Diesem liegt die Annahme zugrunde, dass Unternehmen die ,sourcing“-Entscheidung vor dem Hintergrund der Generierung von Wettbewerbsvorteilen gegenüber Konkurrenten beurteilen. Auch dieser Begründungszusammenhang konnte sich nicht bestätigen, obwohl sich die Hypothese des positiven Zusammenhangs zw ischen „human asset specificity“ und strategischer Bedeutung der IV-Funktionen in allen Modellen (bis auf Systembetrieb / Rechenzentrum) als zutreffend erwies. Bereits in einer 1991 durchgeführten Studie kommt Krcmar [Krcm92] zu dem Urteil, dass das Bewusstsein der mittelständischen DV-Leiter um die strategische Relevanz der IV gering ausgeprägt ist. In einer fünf Jahre später von Spitta [Spit98] durchgeführten Untersuchung im Mittelstand, die sich auf das IV-Controlling bezog, wird deutlich, dass Unternehmen zwar dessen Wichtigkeit betonen, jedoch nichts zu dessen sichtbarer Umsetzung unternehmen. Ähnlich verhält es sich offensichtlich mit der Einschätzung von Spezifität und strategischer Bedeutung der einzelnen IV-Funktionen. Zwar wird die Bedeutung und der Zusammenhang beider Kriterien erkannt, sie finden allerdings in strategischen Entscheidungen wie dem Outsourcing der IV wenig Berücksichtigung. In der bislang einzigen mittelstandsbezogenen empirischen Untersuchung in den USA stellt Borchers [Borc96] ebenfalls keinen Zusammenhang zwischen dem Konstrukt 
der IV als Kernkompetenz und dem Grad des IV-Outsourcing fest, während in der originären Untersuchung von Loh [Loh93], dessen Untersuchungsobjekte sich aus den 500 größten USamerikanischen Unternehmen zusammensetzen, eine starke negative Beziehung festgestellt wird. $^{4}$

Ein relativ starker signifikanter positiver Zusammenhang konnte bei allen Funktionen - bis auf die Funktionen Telekommunikation / Netzwerke sowie Planung und Management - zwischen Ressourcendefiziten gegenüber externen Anbietern und dem Grad des IV-Outsourcing festgestellt werden. Bereits 1991 haben Rumelt et al. [RuST91, S. 19] mit Blick in die Zukunft die Auffassung vertreten, dass Marktkontrakte nicht nur dann in Betracht gezogen werden, wenn Märkte als ineffizient eingestuft werden, sondern auch oder eher dann, wenn Unternehmen bzw. Organisationen selbst bei der adäquaten Aufgabenerfüllung „versagen“. Diese Sichtweise scheint in der vorliegenden Untersuchung die dominierende Stellung einzunehmen. Nicht potenzielle Ineffizienzen externer Dienstleister sind ausschlaggebend für die „make-or-buy“-Entscheidung, sondern die Beurteilung der eigenen Ressourcen und Fähigkeiten im Verhältnis zum Anbietermarkt. Diese Beobachtung lässt auch den negativen Zusammenhang zwischen der Anzahl externer Anbieter und dem Grad des Outsourcing als weniger unplausibel erscheinen. Es lässt sich argumentieren, dass KMU trotz einer geringen Anzahl geeigneter IV-Dienstleister ihre Anwendungsentwicklung, ihren Systembetrieb oder hren Help Desk auslagern, da für sie der Aspekt der Behebung der Ressourcendefizite mit Hilfe externer Anbieter gegenüber der Gefahr der Ressourcenabhängigkeit im Verhältnis zum IVDienstleister überwiegt. Kritiker der Transaktionskostentheorie argumentieren zudem, dass die Annahme, die Gefahr opportunistischen Verhaltens könne innerhalb der Unternehmung durch das Autortitätsprinzip und dementsprechend effizientere Kontrollmechanismen besser unterbunden werden, nicht aufrechterhalten werden könne [GhIn96]. Diese Auffassung findet - wiederum mit Ausnahme der Funktionen Telekommunikation / Netzwerke sowie Planung und Management - implizit Bestätigung durch den positiven signifikanten Zusammenhang zwischen der Schwierigkeit der Messbarkeit der Aufgabenerfüllung der einzelnen IVFunktionen und dem Anteil des IV-Outsourcing. Offensichtlich werden Messschwierigkeiten innerhalb der Unternehmung als Defizite aufgefasst, die von einem externen Anbieter eher behoben werden können als innerhalb der Unternhemensgrenzen. Sie führen demnach zu einem erhöhten IV-Outsourcing. Diese Ergebnisse konnten auch schon bei Heinzl [Hein93] bezogen auf die Ausgliederung - nachgewiesen werden. Als Motive der Ausgliederung konnte unter anderem eine erhöhte Steuer- und Kontrollierbarkeit der IV festgestellt werden. Interessant erscheint zudem, dass bei der Funktion Telekommunikation / Netzwerke keine signifi- 
kante Kausalität zwischen Ressourcendefiziten und Outsourcing festgestellt werden konnte. Diese Beobachtung könnte mit dem geringen Erfahrungswissen der KMU mit dieser neueren Technologie zusammenhängen. Ins Bild passt an dieser Stelle, dass Unternehmen entgegen $\mathrm{H} 2 \mathrm{a}+$ trotz einer geringen Anzahl externer Anbieter ein Outsourcing der Funktion Telekommunikation / Netzwerke vorziehen und gleichzeitig diese IV-Funktion umso mehr auslagern je höher der Wandel der Anforderungen und Aufgaben bezogen auf diese infrastrukturelle Teilfunktion eingeschätzt wird. Offenbar findet hier eine Auslagerung als unreflektierte Anpassung an eine unsichere Umweltsituation statt, die durch eine hohe Innovationskraft des Marktes hervorgerufen wird. Es handelt sich um sogenannte „enabling technologies“, deren Adaption unumgänglich erscheint, um nicht an Wettbewerbsfähigkeit zu verlieren [Beij96, S. $311]$.

Bei der Betrachtung des aggregierten Modells aller IV-Funktionen fällt der moderat ausgeprägte positive Zusammenhang zwischen der Machtposition des IV-Bereichs und dem Grad der Auslagerung der Teilfunktionen auf. Diese von der ursprünglichen Hypothese (H5-) abweichende Beobachtung ist möglicherweise auf die mittelstandsspezifische Situation zurückzuführen. Die deskriptiven Daten haben aufgezeigt, dass die personale und finanzielle Ressourcenausstattung der Informationsverarbeitung in allen untersuchten Unternehmen als gring einzustufen ist. Daher macht es nur bedingt Sinn, den Machtaspekt allein auf die Situation innerhalb der Unternehmung zu beziehen. Vielmehr muss eine mögliche mittelstandsspezifische Veränderung der Machtsituation im Falle einer Auslagerung der IV berücksichtigt werden. Bei einer durchschnittlichen Mitarbeiterzahl von nur fünf Angestellten innerhalb der IV sind die Karriere-, Gehalts- und Weiterentwicklungpfade der IV-Mitarbeiter begrenzt. Es lässt sich daher argumentieren, dass Mitarbeiter im Rahmen eines Beschäftigungsverhältnisses bei einem externen Dienstleister in der Regel ein attraktiveres Unternehmensumfeld vorfinden und dementsprechend eine positive Einstellung zum IV-Outsourcing mitbringen. Gleichsam wird die Machtposition der IV-Abteilung im Falle eines Outsourcing nicht geschwächt, sondern eher gestärkt, da die Koordinationsfunktion eines oder mehrerer externer IVDienstleister in den Vordergrund rückt.

Ins Bild passt diesbezüglich der geringe Anteil der Auslagerung der IV-Funktion Planung und Management. Es fällt auf, dass der Erklärungsgehalt des Modells bezogen auf diese Funktion sehr gering ausfällt. Offensichtlich sind für den Verbleib dieser Funktion innerhalb der Unternehmensgrenzen Aspekte ausschlaggebend, die außerhalb des Erklärungsgehalts des Modells liegen. Insbesondere Aspekte der Koordination und Kontrolle gegenüber einem externen 
Dienstleister sowie die Aufrechterhaltung von Potenzialen zur eigenen Akzentuierung und Richtungsvorgabe in der Informationsverarbeitung könnten sich als relevant erweisen.

\section{Fazit und Ausblick}

Der vorliegende Beitrag beleuchtet die Situation des Outsourcing der Informationsverarbeitung in einer mittelstandsspezifischen Branche. Die wichtigsten Ergebnisse sollen hier nochmal zusammengefasst werden:

- Insgesamt kann der Anteil des Outsourcing der IV in KMU als gering eingestuft werden.

- Die Funktionen der IV werden in KMU selektiv, d.h. in unterschiedlichem Maße ausgelagert. Während die Anwendungsentwicklung den höchsten Anteil ausmacht, verbleibt die Planung und das Management weitgehend innerhalb der Unternehmensgrenzen.

- Die Einschätzung interner Ressourcendefizite gegenüber externen Dienstleistern bildet die dominierende Determinante zur Erklärung des unterschiedlichen Grades der Auslagerung von Funktionen der Informationsverarbeitung.

- Demgegenüber scheinen Rationalitätskalküle wie die Berücksichtigung von Transaktionskosten und die Einschätzung der strategischen Bedeutung der IV in den Hintergrund zu rücken.

Die Ergebnisse dienen dazu, der Praxis im Sinne der „critical social theory“ einen Spiegel vorzuhalten, der ihre eigene Verhaltensweise kritisch reflektiert [Lee99, S. 31]. Die Ergebnisse verstärken den bereits in früheren empirischen mittelstandsspezifischen Studien gewonnenen Eindruck, dass KMU organisatorischen und strategischen Fragestellungen der IV keine allzu große Relevanz beimessen. Diese Sichtweise könnte sich mittelfristig als kritisch erweisen. Als alarmierend ist einzustufen, dass mittelständische Unternehmen bei einer geringen Anzahl von Anbietern und bei eigenen Schwierigkeiten in der Beurteilung der Aufgabenerfüllung der einzelnen Funktionen der IV einen hohen Auslagerungsgrad aufweisen. Es besteht die Gefahr, dass sich Mittelständler in diesen Fällen in eine unreflektierte Abhängigkeitsposition gegenüber einem externen Dienstleister begeben.

Die Studie weist Limitationen auf, die Anknüpfungspunkte für weiterführende Forschungsarbeiten bieten:

- Es wurde nur eine spezifische Branche des Mittelstandes betrachtet. Es gilt zu erforschen, inwieweit die Ergebnisse auf andere Branchen übertragbar sind und ob es Abweichungen im IV-Outsourcingverhalten gegenüber Großunternehmen in Deutschland gibt. ${ }^{5}$ Eine in- 
terkulturelle Studie könnte Aufschlüsse darüber geben, ob das Outsourcingverhalten im deutschen Sprachraum signifikant verschieden von anderen Kulturräumen ist.

- Die funktionale Aufspaltung der IV in 5 Funktionen vernachlässigt die Tatsache, dass diese wiederum in Subfunktionen aufgespalten werden können. Die Aufspaltung könnte z.B. bei der Anwendungsentwicklung die unterschiedlichen Entwicklungsstadien oder eine Aufgabenaufteilung in einzelne Module mit unterschiedlichen Anforderungen widerspiegeln [AlSa95; HeNS96].

- Die Messinstrumentarien des Unsicherheitskonstrukts haben sich als äußerst heterogen erwiesen. In zukünftigen Studien wären die einzelnen Indikatoren als separate Konstrukte zu behandeln und dementsprechend unterschiedliche Begründungszusammenhänge abzuleiten. Für jedes Konstrukt wären mindestens drei Indikatoren zur Messung heranzuziehen [Chin98b].

- In der vorliegenden Arbeit wurden allein Beziehungen auf der Makroebene betrachtet. Jeder dieser Beziehungen liegen Verhaltens- und Einstellungsannahmen auf der Mikrobzw. Individualebene zu Grunde. Diese wären möglicherweise als Konstrukte zu operationalisieren und deren Einfluss auf die Modellstruktur als Intermediatoren oder Moderatoren der Beziehungen auf der Makroebene zu hypothetisieren. Im Rahmen der Transaktionskostentheorie könnte die Messung des Opportunismus oder der opportunistischen Verhaltensweise im Sinne der Verhaltensunsicherheit sowie die Einbeziehung des Vertrauens die Limitationen des neoklassischen methodischen Individualismus beheben [GhIn96; Noor96]. Bei der Erforschung der Beziehungen auf der Individualebene könnte sich ein exploratives Forschungsdesign unter Anwendung der Erhebungsmethodik strukturierter Interviews als zunächst vorteilhaft erweisen.

Abschließend ist festzuhalten, dass die Frage des Outsourcing der IV im Mittelstand nicht weniger spannend ist als in Großbetrieben. Es bleibt nur zu hoffen, dass mittelständische Betriebe sich Instrumentarien und Untersuchungen dieser Art in Zukunft stärker öffnen.

\section{Anmerkungen}

${ }^{1}$ Einer Marktstudie von IDC [IDC99] zufolge betrug das Marktvolumen des IV-Outsourcing in den USA im Jahr 1996 insgesamt 13 Mrd. US\$ und stieg 1998 auf bereits 20,5 Mrd. US\$ an. Bis zum 
Jahr 2003 wird ein jährliches Wachstum von 13,1 \% prognostiziert, was einem Marktvolumen von 38 Mrd. US\$ in 2003 entspricht. Ein Blick auf die Entwicklung des Outsourcingmarktes in Deutschland belegt, dass das Marktvolumen im Jahr 1998 insgesamt 1,7 Mrd. US\$ gegenüber 1,3 Mrd. US\$ in 1996 betrug [IDC98]. Dies entspricht einer Relation USA : Deutschland von 12:1, die sich unter Berücksichtung des Verhältnisses der Bruttosozialprodukte und der anteiligen ITAusgaben (4:1) auf eine Relation von 3:1 relativiert [OECD00].

${ }^{2}$ Für einen Überblick über bisherige Arbeiten zum Outsourcing der IV sei ebenfalls auf Dibbern et al. $[\mathrm{DiGH}]$ verwiesen.

${ }^{3}$ Die gleiche Aggregation wurde von Poppo und Zenger [PoZe98, S. 862] in ihrer empirschen Untersuchung bezogen auf neun verschiedene IV-Funktionen vorgenommen: ,...the core sample was nine IS functions across 152 companies for a total sample of 1368 information service exchanges." Es sei allerdings kritisch angemerkt, dass es sich nicht um unabhängige Stichproben handelt, da die Fragen bezogen auf jede der IV-Funktionen jeweils nur von einer Person innerhalb der Unternehmung beantwortet werden.

${ }^{4}$ Borchers [Borc96, S. 86] führt an: "The concept of core competencies, while frequently mentioned in management literature and embraced in the Fortune 500 firms Loh worked with, may well be a prescription that managers in the medium sized manufacturing firms studied here have not yet come to understand and employ.“

${ }^{5}$ Eine von Alpar und Ein-Dor [AlEi91] durchgeführte Studie im US-amerikanischen Mittelstand zeigt auf, dass deren Bedürfnisse von denen großer Unternehmen abweichen. Während sich KMU auf die Qualität und Zuverlässigkeit der IV fokussieren, legen Großunternehmen das Hauptaugenmerk auf Planung und Management.

\section{Literatur}

[AlEi91] Alpar, P.; Ein-Dor, P.: Major IS Concerns of Entrepreneurial Organizations. In: Information \& Management, 20(1991), S. 1-11.

[A1Sa95] Alpar, P.; Saharia, A. N.: Outsourcing Information System Functions: An Organization Economics Perspective. In: Journal of Organizational Computing, 5(1995)3, S. 197-217.

[ASHS97] Apte, U. M.; Sobol, M. G.; Hanaoka, S.; Shimada, T.; Saarinen, T.; Salmela, T.; Vepsalainen, A. P. J.: IS Outsourcing Practices in the USA, Japan and Finnland: A Comparative Study. In: Journal of Information Technology, 12(1997), S. 289-304. 
[Beij96] Beije, P. R.: Transaction Costs and Technological Learning. In: J. Groenewegen (Hrsg.): Transaction Cost Economics and Beyond. Kluwer Academic Publishers, Boston, Dordrecht, London 1996, S. 309-326.

[Borc96] Borchers, A. S.: Information Technology Outsourcing: A Test of Organizational Economic, Strategic and Political Models, Ph. D. Dissertation, Nova Southeastern University, 1996.

[Chin98a] Chin, W. W.: Exploratory Structural Equation Modeling. Structural Equation Modeling in IS Research: ISWorld Net Virtual Meeting Center at Temple University, http://interact.cis.temple.edu/ vmc (click on "guest"), Abruf am 1998-11-04.

[Chin98b] Chin, W. W.: Issues and Opinion on Structural Equation Modeling. In: Management Information Systems Quarterly, 22(1998b)1, S. VII-XVI.

[Chin98c] Chin, W. W.: The Partial Least Squares Approach for Structural Equation Modeling. In: G. A. Marcoulides (Hrsg.): Modern Methods for Business Research. Lawrence Erlbaum Associates, Hillsdale 1998c, S. 295-336.

[ChNe99] Chin, W. W.; Newsted, P. R.: Structural Equation Modeling: Analysis with Small Samples Using Partial Least Squares. In: R. Hoyle (Hrsg.): Statistical Strategies for Small Sample Research. Sage Public ations, 1999, S. 307-341.

[CIRo91] Clemons, E. K.; Row, M. C.: Sustaining IT Advantage: The Role of Structural Differences. In: MIS Quarterly, 15(1991)3, S. 275-292.

[Conn91] Conner, K. R.: A Historical Comparison of Resource-Based Theory and Five Schools of Thought Within Industrial Organization Economics: Do We Have a New Theory of the Firm? In: Journal of Management, 17(1991)1, S. 121-154.

[CyMa63] Cyert, R. M.; March, J. G.: A Behavioral Theory of the Firm. Prentice-Hall, Englewood Cliffs, N.J., 1963.

[Dear87] Dearden, J.: The Withering Away of the IS Organization. In: Sloan Management Review, 28(1987)4, S. 87-91.

[Dems91] Demsetz, H.: The Theory of the Firm Revisited. In: O. E. Williamson und S. G. Winter (Hrsg.): The Nature of the Firm - Origins, Evolutions, and Development. New York, Oxford 1991, S. $159-178$.

[Dern97] Dernbach, W.: IT-Ausgründung - ein Schuß nach hinten. In: Computerwoche, 4(1997), S. $14-16$. 
[DiGH] Dibbern, J.; Güttler, W.; Heinzl, A.: Die Theorie der Unternehmung als Erklärungsansatz für das selektive Outsourcing der Informationsverarbeitung. Erscheint in: Zeitschrift für Betriebswirtschaftslehre.

[DiCB89] Dierickx, I.; Cool, K.; Barney, J. B.: Asset Stock Accumulation and Sustainability of Competitive Advantage; Comment; Reply. In: Management Science, 35(1989)12, S. 1504-1514.

[Forn89] Fornell, C.: The Blending of Theoretical Empirical Knowledge in Structural Equations with Unobservables. In: H. Wold (Hrsg.): Theoretical Empiricism: A General Rationale for Scientific Model-Building. Paragon House, New York 1989, S. 153-174.

[Foss96] Foss, N. J.: Knowledge-Based Approaches to the Theory of the Firm: Some Critical Comments. In: Organization Science, 7(1996)5, S. 470-476.

[GhIn96] Ghoshal, S.; Insead, P. M.: Bad for Practice: A Critique of the Transaction Cost Theory. In: Academy of Management Review, 21(1996)1, S. 13-47.

[Gran91] Grant, R. M.: The Resource-Based Theory of Competitive Advantage: Implications for Strategy Formulation. In: California Management Review, 33(1991)3, S. 114-135.

[GrCT94] Grover, V.; Cheon, M. J.; Teng, J. T. C.: A Descriptive Study on the Outsourcing of Information Systems Functions. In: Information \& Management, 27(1994)1, S. 33-44.

[Hein69] Heinrich, L.: Zur Frage "Eigenfertigung oder Fremdbezug" bei der Informationsverarbeitung. In: Schmalenbachs Zeitschrift für betriebswirtschaftliche Forschung, 10/11(1969), S. 676688.

[Hein93] Heinzl, A.: Die Ausgliederung der betrieblichen Datenverarbeitung : eine empirische Analyse der Motive, Formen und Wirkungen. 2. Aufl., Poeschel, Stuttgart 1993.

[Hein96] Heinzl, A.: Die Evolution der betrieblichen DV-Abteilung: eine lebenszyklustheoretische Analyse. Physica, Heidelberg 1996.

[HeSt93] Heinzl, A.; Stoffel, K.: Die Ausgliederung der betrieblichen Informationsverarbeitung. In: A. Heinzl und J. Weber (Hrsg.): Alternative Organisationskonzepte der betrieblichen Datenverarbe itung. Stuttgart 1993, S. 173-214.

[HeNS96] Heisekanen, A.; Newman, M.; Similä, J.: Software Contracting: A Process Model Approach. International Conference on Information Systems, 1996, S. 51-62.

[IDC98] IDC.: European Outsourcing Markets and Trends, 1996-2002. International Data Corporation, \#P04E, by M. Lucacs, 1998. 
[IDC99] IDC.: U.S. and Worldwide Outsourcing Markets and Trends, 1998-2003. International Data Corporation, \#19322, by C. Murphy, S. Ker and S. Chen, 1999.

[Knud95] Knudsen, C.: Theories of the Firm, Strategic Management, and Leadership. In: C. A. Montgomery (Hrsg.): Resource-Based and Evolutionary Theories of the Firm: Towards a Synthesis. Boston, Dordrecht, London 1995, S. 179-217.

[Krcm92] Krcmar, H.: Leise Töne im Information Management des Mittelstands. In: Information Management, 7(1992)4, S. 79-83.

[Krcm97] Krcmar, H.: Informationsmanagement. Springer, Berlin u. a. 1997.

[LaHi93] Lacity, M. C.; Hirschheim, R.: Information Systems Outsourcing : Myths, Metaphors, and Realities. Wiley, Chichester, New York 1993.

[LWFi95] Lacity, M. C.; Willcocks, L. P.; Feeny, D. F.: IT Outsourcing: Maximize Flexibility and Control. In: Harvard Business Review, May-June(1995), S. 84-93.

[LaWF96] Lacity, M. C.; Willcocks, L. P.; Feeny, D. F.: The Value of Selective IT Sourcing. In: Sloan Management Review, 37(1996)3, S. 13-25.

[Lang95] Langlois, R. N.: Capabilities and Coherence in Firms and Markets. In: C. A. Montgomery (Hrsg.): Resource-Based and Evolutionary Theories of the Firm: Towards a Synthesis. Boston, Dordrecht, London 1995, S. 71-100.

[Lee99] Lee, A. S.: Rigor and relevance in MIS research: Beyond the approach of positivism alone. In: MIS Quarterly, 23(1999)1, S. 29-33.

[LeBW97] Lee, B.; Barua, A.; Whinston, A. B.: Discovery and representation of casual relationships in MIS research: A methodological framework. In: MIS Quarterly, 21(1997)1, S. 109-136.

[Loh93] Loh, L. Y. K.: The Economics and Organization of Information Technology Governance: Sourcing Strategies for Corporate Information Infrastructure, Ph. D. Dissertation, Massachusetts Institute of Technology, 1993.

[MaFB95] Mata, F. J.; Fuerst, W. L.; Barney, J. B.: Information technology and sustained competitive advantage: A resource-based analysis. In: MIS Quarterly, 19(1995)4, S. 487-505.

[MeKn98] Mertens, P.; Knolmayer, G.: Organisation der Informationsverarbeitung: Grundlagen Aufbau - Arbeitsteilung. 3. Aufl., Wiesbaden 1998. 
[Noor96] Noorderhaven, N. G.: Opportunism and Trust in Transaction Cost Economics. In: J. Groenewegen (Hrsg.): Transaction Cost Economics and Beyond. Kluwer Academic Publishers, Boston, Dordrecht, London 1996, S. 105-128.

[OECD00] OECD.: Information Technolgy Outlook: 2000. Paris, 2000.

[Pfef81] Pfeffer, J.: Power in organizations. Pitman Pub., Marshfield, Mass. 1981.

[PoZe98] Poppo, L.; Zenger, T.: Testing Alternative Theories of the Firm: Transaction Cost, Knowledge-Based, and Measurement Explanations for Make-or-Buy Decisions in Information Services. In: Strategic Management Journal, 19(1998), S. 853-877.

[RuST91] Rumelt, R. P.; Schendel, D.; Teece, D. J.: Strategic Management and Economics. In: Strategic Management Journal(1991), S. 5-29.

[SoAp95] Sobol, M. G.; Apte, U.: Domestic and Global Outsourcing Practices of America's most Effective IS Users. In: Journal of Information Technology, 10(1995), S. 269-280.

[Spit98] Spitta, T.: IV-Controlling in mittelständischen Industrieunternehmen - Ergebnisse einer empirischen Studie. In: Wirtschaftsinformatik, 5(1998), S. 424-433.

[TRDW94] Teece, D. J.; Rumelt, R. P.; Dosi, G.; Winter, S. G.: Understanding Corporate Coherence: Theory and Evidence. In: Journal of Economic Behavior and Organization, 23(1994), S. 1-30.

[Will75] Williamson, O. E.: Markets and Hierarchies, Analysis and Antitrust Implications : A Study in the Economics of Internal Organization. Free Press, New York 1975.

[Will81] Williamson, O. E.: The Economics of Organization: The Transaction Cost Approach. In: American Journal of Sociology, 87(1981)3, S. 548-577.

[Will85] Williamson, O. E.: The Economic Institutions of Capitalism : Firms, Markets, Relational Contracting. Free Press, Collier Macmillan, New York, London 1985.

[Will90] Williamson, O. E.: Transaction Cost Economics. In: R. Schmalensee und R. D. Willig (Hrsg.): Handbook of Industrial Organization. Amsterdam, New York, Oxford, Tokyo 1990, S. 135-182.

[Wold89] Wold, H.: Introduction to the Second Generation of Multivariate Analysis. In: H. Wold (Hrsg.): Theoretical Empiricism: A General Rationale for Scientific Model-Building. Paragon House, New York 1989, S. vii-xl. 
In this paper determinants of IS outsourcing are deduced from transaction cost theory, resourcebased theory and power theory. They are summarized in a theoretical framework which is tested using a sample of 34 small and medium sized firms.

ISSN $\quad 1864-9300$ 